\title{
Hallazgos hematológicos en perros y gatos en Lima, Perú
}

\author{
Hematological findings in dogs and cats in Lima, Peru \\ Raiden Grandía G. ${ }^{1,5}$, Robert Fuentes S. ${ }^{2}$, Joel Pérez P. ${ }^{2}$, Jesús Hernández A. ${ }^{2}$, \\ Melanie Castillo E. ${ }^{2}$, Willian Anicama A. ${ }^{2}$, Jacqueline Caballero C. ${ }^{2}$, Lud Rojas C. ${ }^{2}$, \\ Ivon Galindo J. ${ }^{2}$, Leiner Díaz M. ${ }^{3}$, Rigoberto Fimia-Duarte ${ }^{4}$
}

\section{Resumen}

El objetivo del estudio fue realizar una evaluación hematológica a perros y gatos en Lima, Perú. Se colectaron 460 muestras de sangre (410 perros y 50 gatos) entre diciembre de 2017 y mayo de 2018. Los frotis se colorearon con Wright y las láminas de reticulocitos con Azul de cresil brillante. Se realizó estadística descriptiva para variables cuantitativas en las series eritroide, mieloide y linfoide, así como el análisis de frecuencias de variables cualitativas entre especies indicadoras de tipo de anemia y leucemia, capacidad de regeneración medular, cambios morfológicos anormales, salida de rango fisiológico y presencia de microorganismos extra e intracelular. Predominaron la anemia normocítica normocrómica ( $23.2 \%$ perro, $10 \%$ gato), anemia megaloblástica ( $5.1 \%$ perro), anemia severa microcítica hipocrómica (4\% gato), leucemia mieloide crónica de neutrófilos $(7.1 \%$ perro, $8 \%$ gato), monocitosis con vacuolización citoplasmática en monocitos (4.9\% perro), trombocitosis severa con agregados plaquetarios (6\% gato), policromatofilia $(6.3 \%$ perro), los dacriocitos (54.4\% perro, 64\% gato), acantocitos ( $11.7 \%$ perro, $40 \%$ gato), macrocitos (14.6\% perro, $6 \%$ gato), leucocitos pequeños (10\% perro, $6 \%$ gato), Cytauxzoon felis (20\% gato), Anaplasma spp ( $0.2 \%$ perro) y Mycoplasma spp ( $0.2 \%$ perro, $2 \%$ gato). Solo se evidenciaron diferencias significativas entre sexos en las plaquetas totales $(\mathrm{p}=0.0087)$ y los eosinófilos $(\mathrm{p}=0.0260)$ siendo mayor en perros machos. Se concluye que la prevalencia de $C$. felis en los gatos estudiados es relativamente baja y existe un riesgo de zoonosis de Anaplasma spp y Mycoplasma spp en los propietarios de animales afectados en Lima, Perú.

Palabras clave: hallazgos hematológicos; perros; gatos; Lima; Perú

\footnotetext{
${ }^{1}$ Centro Nacional para la Producción de Animales de Laboratorio, CENPALAB, La Habana, Cuba

${ }^{2}$ Consultorios Veterinarios Widi, Lima, Perú

${ }^{3}$ Empresa Distribuidora y Comercializadora de Medicamentos, EMCOMED, La Habana, Cuba

${ }^{4}$ Consejo Científico Veterinario, CCV, Villa Clara, Cuba

${ }^{5}$ E-mail: raiden.grandia@gmail.com
}

Recibido: 14 de enero de 2019

Aceptado para publicación: 30 de agosto de 2019 
The aim of this study was to conduct a haematological evaluation of dogs and cats in Lima, Peru. A total of 460 blood samples ( 410 dogs and 50 cats) were collected between December 2017 and May 2018. The smears were stained with Wright and the reticulocytes were stained with Brilliant cresyl blue. Descriptive statistics were performed for quantitative variables in the erythroid, myeloid and lymphoid series, as well as the frequency analysis of qualitative variables between species indicator of type of anaemia and leukaemia, ability of medullary regeneration, abnormal morphological changes, out of physiological range and presence of extra and intracellular microorganisms. The most frequent alterations were normochromic normocytic anaemia (23.2\% dog, 10\% cat), megaloblastic anaemia ( $5.1 \% \mathrm{dog})$, severe hypochromic microcytic anaemia (4\% cat), neutrophilic chronic myeloid leukaemia ( $7.1 \% \mathrm{dog}, 8 \% \mathrm{cat})$, monocytosis with cytoplasmic vacuolization in monocytes $(4.9 \% \mathrm{dog})$, severe thrombocytosis with platelet aggregates ( $6 \%$ cat), polychromatophilia (6.3\% dog), dacrocytes (54.4\% dog, 64\% cat), acanthocytes (11.7\% dog, $40 \%$ cat), macrocytes (14.6\% dog, 6\% cat), small leukocytes (10\% dog, 6\% cat), Cytauxzoon felis (20\% cat), Anaplasma spp (0.2\% dog) and Mycoplasma spp (0.2\% dog, $2 \%$ cat). Only significant differences were observed between sexes in total platelets $(p=0.0087)$ and eosinophils $(\mathrm{p}=0.0260)$ being greater in male dogs. It is concluded that the prevalence of C. felis in the cats studied is relatively low and there is a risk of zoonosis of Anaplasma spp and Mycoplasma spp in the owners of affected animals in Lima, Peru.

Key words: hematological findings; dogs; cats; Lima; Peru

\section{INTRODUCCIÓN}

La observación microscópica de sangre periférica constituye una herramienta de gran importancia en el diagnóstico clínico veterinario. Los signos clínicos de los pacientes no siempre indican un diagnóstico presuntivo evidente, de allí surge la necesidad de exámenes complementarios de laboratorio en una amplia gama de casos.

A nivel mundial, patógenos como Mycoplasma spp, Babesia spp, Anaplasma spp y Cytauxzoon felis son los principales causantes de anemias infecciosas transmitidas por artrópodos en perros y gatos (Spada et al., 2014; El Hamiani-Khatat et al., 2015; Hasiri et al., 2016; Savidge et al., 2016). Anaplasma spp, rickettsia intracelular obligada (eritrocitos, granulocitos y plaquetas) causa en perros la anemia no regenerativa normocítica normocrómica, monocitosis, se- vera trombocitopenia (Allison y Little, 2013; De Tommasi et al., 2014) y anemia hemolítica inmuno-mediada (El HamianiKhatat, 2015), y en el gato es causa de linfopenia (Lappin et al., 2015). Cytauxzoon felis es un protozoo intracelular que causa anemia severa no regenerativa, leucopenia, trombocitopenia y hasta cerca del $100 \%$ de mortalidad en los gatos infectados (Allison y Meinkoth, 2010). Por otra parte, Mycoplasma haemocanis (perro) y Mycoplasma haemofelis (gato) son bacterias con alta afinidad por la superficie eritrocítica, causantes de severas anemias hemolíticas regenerativas, particularmente en animales inmunodeprimidos (Allison y Meinkoth, 2010).

Las leucemias constituyen otras de las afecciones de importancia veterinaria por su alta mortalidad, prevaleciendo la mieloide crónica de neutrófilos en el 10\% de los perros. Se presenta raramente en gatos, pero es co- 
mún en perros de edades medianas y avanzadas, donde aparecen abundantes formas inmaduras (bandas). Con frecuencia está acompañada de una leve anemia arregenerativa normocítica normocrómica y ocasionalmente de trombocitopenia o trombocitosis (Dobson et al., 2006; Weiss y Wardrop, 2010; Marino et al., 2017).

Si bien el estudio de las anemias y leucemias constituyen un potente valor diagnóstico, el examen de la morfología celular de las series eritroide, mieloide y linfoide refuerza la obtención de hallazgos de citotoxicidad que pudieran resultar trascendentales en la eficacia del tratamiento veterinario final (Palmer et al., 2015). En Lima, Perú, se ha percibido un incremento en la tendencia de presentación de anemia en animales de compañía con la publicación de algunos resultados específicos (Bustamante, 2015). Sin embargo, muchos laboratorios clínicos, en su mayoría privados, no han reportado casos de leucemias en años de evaluación. En otro sentido, existen escasos reportes locales que describen ampliamente las alteraciones citomorfológicas, lo cual ha estado condicionado en ocasiones por la capacidad analítica de algunos laboratorios y la necesidad de emitir resultados inmediatos. Basado en estas consideraciones, se trazó como objetivo del presente estudio realizar una evaluación hematológica a perros y gatos en Lima, Perú.

\section{Materiales y Métodos}

\section{Tamaño, Selección y Toma de Muestra}

El estudio se realizó en el Laboratorio Clínico de los Consultorios Veterinarios Widi (WIDI), localizado en el distrito Los Olivos, provincia Lima, Perú. Los animales evaluados provinieron de este distrito así como de distritos aledaños. La zona se encuentra entre 75 y $120 \mathrm{msnm}$, y presentó una temperatura promedio de $24{ }^{\circ} \mathrm{C}$ y humedad relativa de $79 \%$ durante el periodo del estudio.
Se estudiaron 460 animales (410 perros y 50 gatos) entre diciembre de 2017 y mayo de 2018. Los animales eran de ambos sexos y con signos clínicos compatibles con afecciones hematológicas (linfoadenomegalia acompañado de fiebre y vómitos, mucosas gingival y ocular pálidas, letargo, diarreas, hepatoesplenomegalia, taquicardia, taquipnea e inapetencia con adelgazamiento marcado). Los animales fueron inmovilizados mediante el método manual de sujeción para la toma de las muestras (Pérez, 2016). Se tomó $1 \mathrm{ml}$ de sangre con jeringuilla de $5 \mathrm{ml}$ y aguja $21 \mathrm{G}$, por punción de la vena radial con previa antisepsia de la zona de extracción con alcohol al $70 \%$. La sangre fue colectada en tubos de $1.5 \mathrm{ml}$ con EDTA K3 como anticoagulante $\left(\right.$ Microtainer $^{\mathbb{R}}$ ) y procesada dentro de las dos horas de extraídas.

\section{Evaluación Hematológica}

Las variables hematológicas estudiadas se compararon con los rangos fisiológicos de referencia publicados en los manuales veterinarios de hematología y de coagulación del Laboratorio Suiza Vet, Perú (2018) (Cuadro 1).

\section{Serie eritroide}

El hematocrito (Hto) se determinó por centrifugación de la sangre en microcapilares de cristal de $75 \mathrm{~mm}$ a $1200 \mathrm{~g}$ durante $5 \mathrm{~min}$ y el empleo de la escala milimetrada de microhematocrito de Shanghai (Muñoz y Morón, 2005). La hemoglobina ( $\mathrm{Hb}$ ) se calculó a partir del valor del Hto multiplicándolo por el factor 0.33 (la Hb representa aproximadamente entre el 30 y $33 \%$ del Hto) (Forrellat-Barrios et al., 2010). Esta última fórmula se empleó por la ausencia de otras alternativas para su medición en el laboratorio.

El conteo de eritrocitos totales (ET) se realizó en cámara de Neubauer bajo microscopio óptico a 40X. Para ello se depositaron $1990 \mu \mathrm{l}$ de reactivo Hayem en un tubo de cristal, y se añadieron $10 \mu \mathrm{l}$ de sangre total 
Cuadro 1. Valores hematológicos referenciales para perro y gato ${ }^{1}$

\begin{tabular}{|c|c|c|c|}
\hline Serie & Variable & Perro & Gato \\
\hline \multirow[t]{7}{*}{ Eritroide } & Hemoglobina $(\mathrm{g} / \mathrm{dl})$ & $12-18$ & $8-15$ \\
\hline & Hematocrito $(\%)$ & $37-52$ & $30-45$ \\
\hline & Eritrocitos totales $\left(\times 10^{6} / \mathrm{mm}^{3}\right)$ & $5.5-8.2$ & $4.6-10$ \\
\hline & Reticulocitos (\%) & $0.5-1.5$ & $0.1-1.0$ \\
\hline & Volumen corpuscular medio - VCM (fl) & $60-77$ & $40-58$ \\
\hline & Hemoglobina corpuscular media - HCM (pg) & $17-30$ & $12-20$ \\
\hline & $\begin{array}{l}\text { Concentración de hemoglobina corpuscular } \\
\text { media - CHCM }(\mathrm{g} / \mathrm{dl})\end{array}$ & $31-37$ & $29-37$ \\
\hline \multirow{9}{*}{$\begin{array}{l}\text { Mieloide y } \\
\text { linfoide }\end{array}$} & Leucocitos totales $\left(\times 10^{3} / \mathrm{mm}^{3}\right)$ & $9-15$ & $5-14.5$ \\
\hline & Precursores linfoide y mieloide (\%) & 0 & 0 \\
\hline & Neutrófilos bandas (\%) & $0-3$ & $0-3$ \\
\hline & Neutrófilos segmentados (\%) & $60-77$ & $50-60$ \\
\hline & Eosinófilos $(\%)$ & $0-5$ & $2-8$ \\
\hline & Basófilos (\%) & $0-1$ & $0-2$ \\
\hline & Monocitos (\%) & $0-8$ & $0-5$ \\
\hline & Linfocitos $(\%)$ & $13-30$ & $30-35$ \\
\hline & Plaquetas totales $\left(\mathrm{x} 10^{3} / \mathrm{mm}^{3}\right)$ & $175-490$ & $150-600$ \\
\hline
\end{tabular}

${ }^{1}$ Laboratorio Suiza Vet, Perú (2018)

en dilución $1 / 200$, se mezcló suavemente y se dejó reposar durante $5 \mathrm{~min}$. Se montó la muestra en la cámara, se dejó reposar por 3 min, se contaron los cinco cuadrantes del retículo central (central y 4 angulares) y se empleó la fórmula siguiente: células contadas $\times 1 / 1000=\mathrm{ET} \times 10^{6} / \mathrm{mm}^{3}$ (Muñozy Morón, 2005).

El porcentaje relativo de reticulocitos (PRR) se realizó en frotis de sangre periférica con la tinción Azul de cresil brillante (1\%) en microscopio óptico a 100X con aceite de inmersión. Se preparó una mezcla homogénea a partes iguales de sangre total y solución colorante, se dejó reposar 20 min y se ejecutó la extensión en lámina. Se contaron los reticulocitos existentes en 10 campos de aproximadamente 100 células cada uno. El valor se dividió entre 1000 eritrocitos y se multiplicó por 100 para expresarlo en porcentaje. El porcentaje corregido de reticulocitos (PCR) se calculó por la fórmula siguiente:
PRR x (Hto del paciente/Hto máximo fisiológico). Asimismo, el conteo absoluto de estos (CAR) fue: ET x PRR x $10=$ CAR x $10^{3} / \mathrm{mm}^{3}$ (Palomo et al., 2005).

Las constantes corpusculares se determinaron por las fórmulas siguientes: $\mathrm{VCM}=$ Hto $\mathrm{x} 10 / \mathrm{ET}, \mathrm{HCM}=\mathrm{Hb} \times 10 / \mathrm{ET}$ y CHCM $=\mathrm{Hb} \times 100 /$ Hto (Ramis, 2014).

\section{Series mieloide y linfoide}

El recuento total de leucocitos (LT) se realizó en cámara de Neubauer bajo microscopio óptico a 10X. Para ello se depositaron 380 $\mu l$ de reactivo Türk ( $1 \%$ ) en un tubo de cristal, se añadieron $20 \mu$ lde sangre total en dilución $1 /$ 20, se mezcló suavemente y se dejó reposar durante $3 \mathrm{~min}$. Posteriormente se montó la muestra en la cámara, se dejó reposar otros 3 min, se contaron los 4 cuadrantes grandes angulares del retículo y se empleó la fórmula siguiente: células contadas $\times 50 / 1000=\mathrm{LT}$ x $10^{3} / \mathrm{mm}^{3}$ (Muñoz y Morón, 2005). 
El conteo diferencial de leucocitos se realizó en frotis de sangre periférica con la tinción de Wright. Se contaron 100 células en total y se expresaron las cantidades observadas en porcentaje. Se agruparon los precursores mieloide y linfoide para facilitar la separación en la fórmula leucocitaria y poder establecer la comparación con los rangos fisiológicos de referencia (Muñoz y Morón, 2005).

Las plaquetas totales (PT) se determinaron en 10 campos de frotis de sangre periférica con la tinción de Wright en microscopio óptico a 100X con aceite de inmersión. Se aplicó la fórmula siguiente: células contadas $\mathrm{x} 1000=\mathrm{PT} \times 10^{3} / \mathrm{mm}^{3}$ (Muñoz y Morón, 2005).

\section{Morfología celular}

La morfología celular se determinó en frotis de sangre periférica con ambas tinciones en microscopio óptico a 100X con aceite de inmersión. Se evaluaron tamaño, color, forma, segmentación nuclear, granulación citoplasmática, condensación cromatínica, nucléolos, proporción núcleo-citoplasma e inclusiones celulares en las series eritroide, mieloide y linfoide, según fuera aplicable. Asimismo, se verificó la existencia de formas microbianas extra e intracelularmente. Para ello se emplearon los atlas del libro Schalm's veterinary hematology (Weiss y Wardrop, 2010).

\section{Patógenos}

La identificación de los patógenos Anaplasma spp, Cytauxzoon felis y Mycoplasma spp en las muestras de sangre se realizó en frotis de sangre periférica tomando como referencia las imágenes publicadas en los libros de Weiss y Wardrop (2010) y Reagan et al. (2019). En consideración a la limitante de este método de no presentar una elevada sensibilidad diagnóstica se hizo en paralelo la tinción con los colorantes Wright y Azul de cresil brillante para mayor complementariedad en el diagnóstico. Asimis- mo, las muestras fueron observadas por dos analistas con gran experticia y se excluyeron los animales dudosos de los resultados finales.

\section{Criterios de Severidad}

Los criterios de severidad se establecieron según las consideraciones de Weiss y Wardrop (2010) y el Manual Veterinario de Hematología del Laboratorio Suiza Vet de Perú (2018) para cada especie:

- Anemia severa: perro (Hto $<20 \%$ ), gato (Hto $<14 \%)$

- Anemia no regenerativa: perro (CAR $\left.\leq 60 \times 10^{3} / \mathrm{mm}^{3}\right)$, gato $\left(\mathrm{CAR} \leq 50 \times 10^{3} / \mathrm{mm}^{3}\right)$

- Leucocitosis severa: perro y gato (leucocitos totales $\geq 50 \times 10^{3} / \mathrm{mm}^{3}$ )

- Neutrofilia severa: perro y gato (neutrófilos segmentados $\geq 90 \%$ )

- Trombocitosis severa: perro (plaquetas totales $>790 \times 10^{3} / \mathrm{mm}^{3}$ ), gato (plaquetas totales $>900 \times 10^{3} / \mathrm{mm}^{3}$ )

\section{Análisis Estadístico}

Se realizó la estadística descriptiva para las variables cuantitativas en las series eritroide, mieloide y linfoide, así como el análisis de frecuencias de variables cualitativas interespecie indicadoras de tipo de anemia y leucemia, capacidad de regeneración medular, cambios morfológicos anormales, salida de rango fisiológico y presencia de microorganismos extra e intracelulares. La distribución de frecuencias incluyó tanto la absoluta (cantidad de animales observados en cada variable) como la relativa (\% que representó la anterior del total analizado).

Para la estadística inferencial se verificaron si los datos (variables hematológicas) seguían una distribución normal (prueba Kolmogorov-Smirnov) y si cumplían con la homogeneidad de varianza (prueba de Levene). Al no seguir estos una distribución normal, se realizó la prueba no paramétrica Wilcoxon para la comparación de medias intraespecie (hembras $v s$ machos) de las variables cuantitativas en las series eritroide, 
Cuadro 2. Estadística descriptiva de variables en la serie eritroide en perros $(\mathrm{n}=410)$ y gatos $(\mathrm{n}=50)$ con signos clínicos compatibles con afecciones hematológicas (Lima, Perú)

\begin{tabular}{lccccccccc}
\hline Variable & Especie & Media & DE & Varianza & EE & CV $(\%)$ & Mediana & Mín. & Máx. \\
\hline $\mathrm{Hb}(\mathrm{g} / \mathrm{dl})$ & Perro & 13.97 & 3.46 & 11.95 & 0.17 & 24.7 & 14.90 & 3.3 & 22.4 \\
& Gato & 12.15 & 3.07 & 9.42 & 0.43 & 25.3 & 12.90 & 2.6 & 17.8 \\
$\mathrm{Hto}(\%)$ & Perro & 42.32 & 10.47 & 109.7 & 0.52 & 24.8 & 45.00 & 10.0 & 68.0 \\
& Gato & 36.82 & 9.31 & 86.7 & 1.32 & 25. & 39.00 & 8.0 & 54.0 \\
$\mathrm{ET}\left(\times 10^{6} / \mathrm{mm}^{3}\right)$ & Perro & 5.66 & 1.15 & 1.33 & 0.06 & 20.3 & 6.00 & 2.1 & 8.5 \\
& Gato & 6.05 & 1.02 & 1.05 & 0.14 & 16.9 & 6.30 & 2.9 & 7.9 \\
Porcentaje & Perro & 1.30 & 1.24 & 1.54 & 0.06 & 95.5 & 0.90 & 0.1 & 12.3 \\
corregido de ret. & Gato & 1.19 & 1.52 & 2.30 & 0.21 & 127.6 & 0.60 & 0.1 & 8.2 \\
$\mathrm{CAR}\left(\times 10^{3} / \mathrm{mm}^{3}\right)$ & Perro & 91.96 & 91.18 & 8,313 & 4.50 & 99.2 & 65.00 & 5.8 & 969.5 \\
& Gato & 90.35 & 111.87 & 12,514 & 15.8 & 123.8 & 46.05 & 6.2 & 601.4 \\
$\mathrm{VCM}(\mathrm{fl})$ & Perro & 73.90 & 4.88 & 23.84 & 0.24 & 6.6 & 75.30 & 47.6 & 80.2 \\
& Gato & 59.69 & 7.58 & 57.46 & 1.07 & 12.7 & 61.95 & 27.8 & 68.4 \\
$\mathrm{HCM}(\mathrm{pg})$ & Perro & 24.39 & 1.61 & 2.60 & 0.08 & 6.6 & 24.90 & 15.7 & 26.5 \\
& Gato & 19.68 & 2.50 & 6.24 & 0.35 & 12.7 & 20.50 & 9.2 & 22.5 \\
$\mathrm{CHCM}(\mathrm{g} / \mathrm{dl})$ & Perro & 33.00 & 0.04 & 0.00 & 0.00 & 0.1 & 33.00 & 32.8 & 33.3 \\
& Gato & 32.99 & 0.03 & 0.00 & 0.00 & 0.1 & 33.00 & 32.9 & 33.1 \\
\hline
\end{tabular}

DE: Desviación estándar, EE: Error estándar, CV: Coeficiente de variación, Mín.: Mínimo, Máx.: Máximo, Hb: Hemoglobina, Hto: Hematocrito, ET: Eritrocitos totales, Ret.: Reticulocitos, CAR: Conteo absoluto de reticulocitos, VCM: Volumen corpuscular medio, HCM: Hemoglobina corpuscular media, CHCM: Concentración de hemoglobina corpuscular media

mieloide y linfoide. Para el análisis de los resultados se consideró como significación estadística una $\mathrm{p}<0.05$. El programa estadístico utilizado fue el InfoStat v. 2.0.

\section{Resultados y Discusión}

En el Cuadro 2 se aprecia la estadística descriptiva de las variables en estudio (serie eritroide) para perros y gatos que asistieron a consulta médica con diversos signos compatibles con afecciones hematológicas. Las diferencias propias de cada especie estuvieron más marcadas en las medias y medianas del Hto, VCM y la HCM, así como en las medianas del porcentaje corregido y el conteo absoluto de reticulocitos. Independientemente de haber realizado este estudio en animales con signos y síntomas clínicos, las medias globales de las variables analizadas estuvie- ron en su mayoría en el rango fisiológico de referencia, con excepción del porcentaje de reticulocitos y el VCM en los gatos.

En otro sentido, el Cuadro 3 evidencia la estadística descriptiva para las series mieloide y linfoide, particularmente la fórmula leucocitaria, los leucocitos y las plaquetas totales. Se pueden observar mayores diferencias en las medias y medianas de los neutrófilos segmentados, linfocitos y en las plaquetas totales entre ambas especies. Las medias globales de las variables analizadas estuvieron en su gran mayoría dentro del rango fisiológico de referencia aunque fueron animales enfermos. Sin embargo, los valores de los neutrófilos segmentados, leucocitos y las plaquetas totales superaron el límite superior en los gatos evaluados. Asimismo, se apreció en ambas especies la salida del rango del porcentaje de neutrófilos bandas y de los precursores mieloide y linfoide. 
Cuadro 3. Estadística descriptiva de variables en las series mieloide y linfoide en perros $(\mathrm{n}=410)$ y gatos $(\mathrm{n}=50)$ con signos clínicos compatibles con afecciones hematológicas (Lima, Perú)

\begin{tabular}{lccccccccc}
\hline Variable & Especie & Media & DE & Varianza & EE & CV (\%) & Mediana & Mín. & Máx. \\
\hline LT & Perro & 14.53 & 7.88 & 62.10 & 0.39 & 54.2 & 13 & 1.6 & 65.9 \\
$\left(\times 10^{3} / \mathrm{mm}^{3}\right)$ & Gato & 16.71 & 11.30 & 127.74 & 1.60 & 67.6 & 13 & 5.0 & 58.8 \\
Precursores & Perro & 0.20 & 1.05 & 1.11 & 0.05 & 514.7 & 0 & 0 & 9.0 \\
linfoides (\%) & Gato & 0.26 & 0.92 & 0.85 & 0.13 & 354.5 & 0 & 0 & 6.0 \\
Precursores & Perro & 0.17 & 1.68 & 2.82 & 0.08 & 969.4 & 0 & 0 & 30.0 \\
mieloides (\%) & Gato & 0.02 & 0.14 & 0.02 & 0.02 & 707.1 & 0 & 0 & 1.0 \\
Neut. bandas & Perro & 0.57 & 2.07 & 4.27 & 0.10 & 365.2 & 0 & 0 & 23.0 \\
$(\%)$ & Gato & 0.36 & 1.03 & 1.05 & 0.15 & 284.8 & 0 & 0 & 5.0 \\
Neut. segm. & Perro & 72.45 & 14.09 & 198.57 & 0.70 & 19.5 & 75 & 20 & 96.0 \\
$(\%)$ & Gato & 67.78 & 18.87 & 355.89 & 2.67 & 27.8 & 69 & 32 & 99.0 \\
Eosinófilos & Perro & 2.42 & 3.40 & 11.56 & 0.17 & 140.2 & 1 & 0 & 20.0 \\
$(\%)$ & Gato & 4.44 & 4.67 & 21.84 & 0.66 & 105.3 & 3 & 0 & 17.0 \\
Basófilos & Perro & 0.04 & 0.24 & 0.06 & 0.01 & 542.6 & 0 & 0 & 3.0 \\
$(\%)$ & Gato & 0.08 & 0.27 & 0.08 & 0.04 & 342.6 & 0 & 0 & 1.0 \\
Monocitos & Perro & 5.47 & 4.31 & 18.55 & 0.21 & 78.7 & 5 & 0 & 31.0 \\
$(\%)$ & Gato & 3.40 & 2.32 & 5.39 & 0.33 & 68.3 & 4 & 0 & 9.0 \\
Linfocitos & Perro & 18.67 & 13.06 & 170.50 & 0.64 & 69.9 & 16 & 0 & 76.0 \\
$(\%)$ & Gato & 23.66 & 16.72 & 279.45 & 2.36 & 70.7 & 21 & 1.0 & 60.0 \\
PT & Perro & 468.32 & 298.12 & $88,876.32$ & 14.72 & 63.7 & 400 & 20.0 & 3,000 \\
$\left(\times 10^{3} / \mathrm{mm}^{3}\right)$ & Gato & 611.62 & 304.66 & $92,819.51$ & 43.09 & 49.8 & 575 & 190 & 1,500 \\
\hline
\end{tabular}

DE: Desviación estándar, EE: Error estándar, CV: Coeficiente de variación, Mín.: Mínimo, Máx.: Máximo, LT: Leucocitos totales, Neut.: Neutrófilos, Segm.: Segmentados, PT: Plaquetas totales

El Cuadro 4 muestra la frecuencia de presentación del tipo de anemia que manifestaron los animales afectados. Se observó que la anemia normocítica normocrómica tuvo mayor incidencia en los perros $(23.2 \%)$, seguida de la anemia megaloblástica (5.1\%). En este estudio, las formas severas de la anemia tuvieron un menor predominio.

El 90\% de los animales presentaron signos clínicos comunes con la anemia en perros y gatos, principalmente la depresión general, fatiga, desinterés en las actividades cotidianas y palidez en las mucosas, tal y como lo señalan Gholipour (2016) y Ewing (2018). Por otro lado, la deficiencia congénita de vitamina B12 en algunas razas de perros, además de la anemia, afecta el creci- miento de los animales afectados, denotando un signo común a otras afecciones no hematopoyéticas. Así ocurre en presencia de la anemia por deficiencia de hierro, donde su diagnóstico puede apoyarse en la observación microscópica de microcitosis e hipocromasia, así como en las desencadenadas por daños directos sobre la médula ósea (quimioterapia) y sobre el riñón (fallo en la producción de eritropoyetina) donde aparece una sintomatología inespecífica (Gholipour, 2016; Ewing, 2018).

Existen otros tipos de anemia en perros y gatos, que aunque no presentan signos clínicos concretos de una determinada afección de la serie eritroide, pueden ser diagnosticadas de manera más confiable en el laborato- 
Cuadro 4. Análisis de frecuencia de variables relacionadas en la serie eritroide en perros $(n=410)$ y gatos $(\mathrm{n}=50)$ con signos clínicos compatibles con afecciones hematológicas (Lima, Perú)

\begin{tabular}{llccc}
\hline Variable & Hallazgo & Especie & FA & FR (\%) \\
\hline Hematocrito & Anemia normocítica & Perro & 95 & 23.2 \\
Volumen corpuscular medio & normocrómica & Gato & 5 & 10.0 \\
(VCM) & Anemia severa normocítica & Perro & 3 & 0.7 \\
Hemoglobina corpuscular media & normocrómica & Gato & 0 & 0.0 \\
(HCM) & Anemia severa microcítica & Perro & 9 & 2.2 \\
Concentración de hemoglobina & normocrómica & Gato & 0 & 0.0 \\
corpuscular media (CHCM) & Anemia severa microcítica & Perro & 2 & 0.5 \\
& hipocrómica & Gato & 2 & 4.0 \\
& & & & \\
Hematocrito & Anemia megaloblástica & Perro & 21 & 5.1 \\
Megalocito & & Gato & 0 & 0.0 \\
Policromatofilia & & & & \\
Hematocrito & Anemia regenerativa & Perro & 50 & 38.5 \\
Conteo absoluto de & Gato & 3 & 42.9 \\
reticulocitos (CAR) & Anemia severa regenerativa & Perro & 9 & 6.9 \\
& & Gato & 1 & 14.2 \\
\hline
\end{tabular}

FA: Frecuencia absoluta, FR: Frecuencia relativa

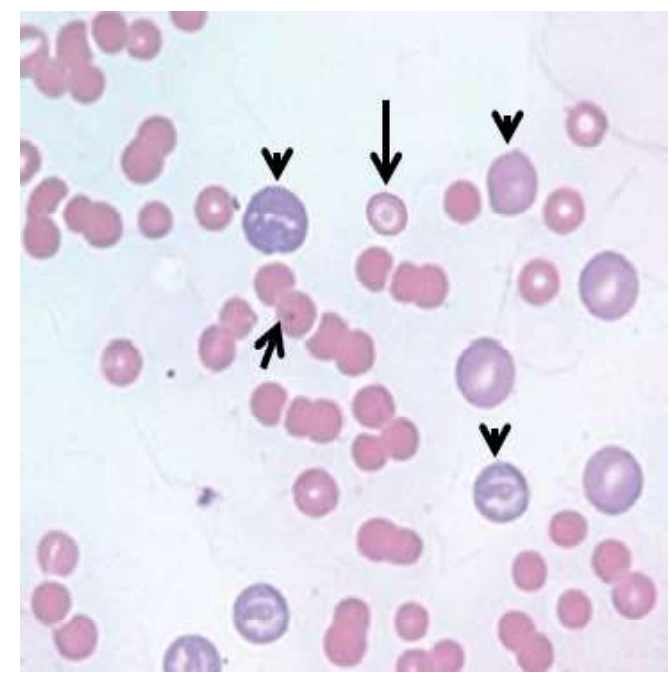

Figura 1. Anemia megaloblástica en perro. Anisocitosis (abundantes megalocitos) y anisocromía (policromatofilia) (cabeza de flecha). Despoblación eritrocítica con severa poiquilocitosis. Eferocito con un cuerpo de Howell-Jolly (flecha corta). Codocito (flecha larga). Tinción de Wright (100X) rio como son las hemolíticas inmuno-mediadas (AHIM) y las inflamatorias. Las AHIM son más comunes en perros que en gatos $\mathrm{y}$ pueden causar en su forma más agresiva hasta un $70 \%$ de mortalidad. En perros se caracterizan por una gran eferocitosis (94\%), trombocitopenia (70\%), coagulación intravascular diseminada (CID) (58\%) y aglutinación eritrocítica (42\%), y son causadas en perros y gatos por infecciones eritrocitarias (Ehrlichia spp, Anaplasma spp, Mycoplasma spp, Babesia spp, Cytauxzoon felis, FeLV), vacunaciones, medicamentos y neoplasias (Weiss y Wardrop, 2010). Según Swann et al. (2016), existe una predisposición importante a sufrir la AHIM en los gatos con edad comprendida entre 2.1 y 5.9 años.

La anemia megaloblástica fue la segunda de mayor presentación en los perros afectados, observándose la presencia de megalocitos y la policromatofilia en todos los 
Cuadro 5. Análisis de frecuencia de variables relacionadas en las series mieloide y linfoide en perros $(n=410)$ y gatos $(n=50)$ con signos clínicos compatibles con afecciones hematológicas (Lima, Perú)

\begin{tabular}{|c|c|c|c|c|}
\hline Variable & Hallazgo & Especie & FA & FR $(\%)$ \\
\hline Leucocitosis severa & \multirow{2}{*}{$\begin{array}{l}\text { Leucemia mieloide crónica de } \\
\text { neutrófilos }\end{array}$} & Perro & 29 & 7.1 \\
\hline $\begin{array}{l}\text { Neutrofilia severa } \\
\text { Morfología anormal }\end{array}$ & & Gato & 4 & 8.0 \\
\hline \multirow{2}{*}{$\begin{array}{l}\text { Vacuolización citoplasmática } \\
\text { en monocitos } \\
\text { Vacuolización citoplasmática } \\
\text { en neutrófilos }\end{array}$} & \multirow{2}{*}{$\begin{array}{l}\text { Vacuolización citoplasmática } \\
\text { en monocitos y neutrófilos }\end{array}$} & Perro & 1 & 0.2 \\
\hline & & Gato & 1 & 2.0 \\
\hline \multirow{2}{*}{$\begin{array}{l}\text { Monocitosis } \\
\text { Vacuolización citoplasmática } \\
\text { en monocitos }\end{array}$} & \multirow{2}{*}{$\begin{array}{l}\text { Monocitosis y vacuolización } \\
\text { citoplasmática en monocitos }\end{array}$} & Perro & 20 & 4.9 \\
\hline & & Gato & 0 & 0 \\
\hline \multirow{2}{*}{$\begin{array}{l}\text { Monocitosis } \\
\text { Núcleo atípico en monocitos }\end{array}$} & \multirow{2}{*}{$\begin{array}{l}\text { Monocitosis y núcleos atípicos } \\
\text { en monocitos }\end{array}$} & Perro & 3 & 0.7 \\
\hline & & Gato & 0 & 0 \\
\hline \multirow{2}{*}{$\begin{array}{l}\text { Vacuolización citoplasmática } \\
\text { en monocitos } \\
\text { Núcleo atípico en monocitos }\end{array}$} & \multirow{2}{*}{$\begin{array}{l}\text { Vacuolización citoplasmática } \\
\text { y núcleos atípicos en } \\
\text { monocitos }\end{array}$} & Perro & 1 & 0.2 \\
\hline & & Gato & 0 & 0 \\
\hline \multirow{2}{*}{$\begin{array}{l}\text { Linfocitosis } \\
\text { Linfocitos reactivos }\end{array}$} & \multirow{2}{*}{$\begin{array}{l}\text { Linfocitosis y linfocitos } \\
\text { reactivos }\end{array}$} & Perro & 2 & 0.5 \\
\hline & & Gato & 0 & 0 \\
\hline \multirow{4}{*}{$\begin{array}{l}\text { Trombocitosis } \\
\text { Macroplaquetas }\end{array}$} & \multirow{2}{*}{$\begin{array}{l}\text { Trombocitosis y } \\
\text { macroplaquetas }\end{array}$} & Perro & 14 & 3.4 \\
\hline & & Gato & 1 & 2.0 \\
\hline & \multirow{2}{*}{$\begin{array}{l}\text { Trombocitosis severa y } \\
\text { macroplaquetas }\end{array}$} & Perro & 10 & 2.4 \\
\hline & & Gato & 0 & 0 \\
\hline \multirow{4}{*}{$\begin{array}{l}\text { Trombocitosis } \\
\text { Agregados plaquetarios }\end{array}$} & \multirow{2}{*}{$\begin{array}{l}\text { Trombocitosis y agregados } \\
\text { plaquetarios }\end{array}$} & Perro & 2 & 0.5 \\
\hline & & Gato & 3 & 6.0 \\
\hline & \multirow{2}{*}{$\begin{array}{l}\text { Trombocitosis severa y } \\
\text { agregados plaquetarios }\end{array}$} & Perro & 3 & 0.7 \\
\hline & & Gato & 3 & 6.0 \\
\hline \multirow{2}{*}{$\begin{array}{l}\text { Macroplaquetas } \\
\text { Agregados plaquetarios }\end{array}$} & \multirow{2}{*}{$\begin{array}{l}\text { Macroplaquetas y agregados } \\
\text { plaquetarios }\end{array}$} & Perro & 1 & 0.2 \\
\hline & & Gato & 0 & 0 \\
\hline
\end{tabular}

FA: Frecuencia absoluta, FR: Frecuencia relativa

casos (Cuadro 4, Figura 1). Aunque la existencia de macrocitos, hipercromasia y policromasia constituyen hallazgos presentes en las anemias regenerativas (Barger, 2010), en ocasiones estos acompañan determinadas condiciones agudas bastante agresivas en esta especie. En este sentido, a diferencia de los gatos (infrecuente), la policromatofilia puede observarse ocasionalmente en los frotis de sangre teñidos con Wright en los perros, pero esta observación debe ser inferior al 1\% (Rizzi et al., 2010). En el presente estudio, su presentación fue cinco veces superior a ese valor fisiológico.

El 31.7\% (130/410) de los perros presentaron anemia (Cuadro 4), donde el 45.4\% fue regenerativa. Asimismo, el 14\% (7/50) 
de los gatos evaluados tuvieron algún tipo de anemia en los cuales el $57.1 \%$ fue regenerativa, lo que indica mayor capacidad de regeneración en los felinos estudiados. Para ello se tomó en consideración los criterios de clasificación del Manual Veterinario de Hematología del Laboratorio Suiza Vet de Perú (2018), donde la anemia se considera no regenerativa en perro cuando el $\mathrm{CAR} \leq 60$ $\mathrm{x} 10^{3} / \mathrm{mm}^{3}$, mientras que en el gato está presente si el CAR $\leq 50 \times 10^{3} / \mathrm{mm}^{3}$.

Es importante añadir que la médula ósea libera a sangre periférica aproximadamente el $1 \%$ de reticulocitos agregados en los perros, y estos maduran en las primeras 24 horas, mientras que los reticulocitos punteados tienen una vida más corta y una cantidad poco significativa. En contraste, existe una baja liberación de agregados $(<0.4 \%)$ en los gatos con una corta vida media (alrededor de 12 horas) en sangre periférica, pero estos se convierten posteriormente en reticulocitos punteados y circulan entre 10-12 días hasta su maduración, representando finalmente el $10 \%$ de los eritrocitos (Christian, 2010).

En el Cuadro 5 se muestran las principales alteraciones de las series mieloide y linfoide, observándose principalmente en la morfología celular en perros, la vacuolización citoplasmática y núcleos atípicos en monocitos y los linfocitos reactivos. Asimismo, predominaron en ambas especies las macroplaquetas, los agregados plaquetarios y la leucemia mieloide crónica de neutrófilos.

La presentación de leucemia mieloide crónica (LMC) de neutrófilos en perros fue de $7.1 \%$, ocurriendo con leucocitosis severa (leucocitos totales $\geq 50 \times 10^{3} / \mathrm{mm}^{3}$ ), neutrofilia severa (neutrófilos segmentados $\geq 90 \%$ ) y neutrófilos maduros pequeños en el $100 \%$ de los casos, además de un $70 \%$ de hipersegmentación nuclear en neutrófilos ( $\geq 5$ lóbulos) y $35 \%$ de neutrófilos bandas gigantes (Cuadro 5, Figura 2). Estos hallazgos coinciden en su mayoría con Dobson et al. (2006),

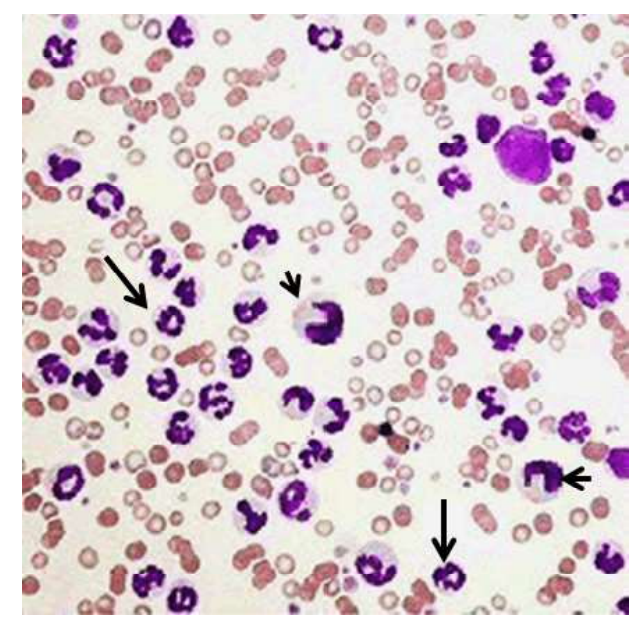

Figura 2. Leucemia mieloide crónica de neutrófilos en perro. Severa neutrofilia con desviación a la izquierda. Neutrófilos bandas gigantes con núcleo amorfo y contenido anormal intracitoplasmático (flecha corta). Neutrófilos pequeños con aspecto maduro y con hipersegmentación nuclear (flecha larga). Abundantes leptocitos, anisocromía y anisocitosis. Tinción de Wright (20X)

quienes plantean que la LMC de neutrófilos se presenta con severa neutrofilia y considerable desviación a la izquierda, así como con cambios atípicos en neutrófilos y presencia de mieloblastos $(\leq 20 \%)$ en médula ósea, lo cual la distingue de una reacción leucemoide a causa de inflamación o infección.

Estos resultados también coinciden con Fine y Tvedten (1999), quienes mencionan la aparición de neutrófilos con hipersegmentación nuclear y neutrófilos bandas gigantes; sin embargo, no coincidieron con la presencia de intensa basofilia citoplasmática. Esta afección se manifiesta con signos recurrentes en los pacientes, pero en muchas ocasiones son asintomáticos, aunque existe con frecuencia hepatoesplenomegalia debido a la infiltración de precursores granulocíticos (Fine y Tvedten, 1999; Dobson et al., 2006). Aquí puede estar presente también la anemia microcítica hipocrómica $(10 \%)$, macrocítica hipocrómica (12.5\%), abundantes esquistocitos y eferocitos pero escasa 
Cuadro 6. Análisis de frecuencia de variables de daño celular en la serie eritroide en perros $(n=410)$ y gatos $(n=50)$ con signos clínicos compatibles con afecciones hematológicas (Lima, Perú)

\begin{tabular}{|c|c|c|c|c|}
\hline Variable & Hallazgo & Especie & FA & FR $(\%)$ \\
\hline \multirow[t]{2}{*}{ Color } & \multirow[t]{2}{*}{ Policromatofilia } & Perro & 26 & 6.3 \\
\hline & & Gato & 0 & 0.0 \\
\hline \multirow[t]{6}{*}{ Tamaño } & \multirow[t]{2}{*}{ Microcito } & Perro & 7 & 1.7 \\
\hline & & Gato & 1 & 2.0 \\
\hline & \multirow[t]{2}{*}{ Macrocito } & Perro & 60 & 14.6 \\
\hline & & Gato & 3 & 6.0 \\
\hline & \multirow[t]{2}{*}{ Megalocito } & Perro & 21 & 5.1 \\
\hline & & Gato & 0 & 0.0 \\
\hline \multirow[t]{18}{*}{ Forma } & \multirow[t]{2}{*}{ Acantocito } & Perro & 48 & 11.7 \\
\hline & & Gato & 20 & 40.0 \\
\hline & \multirow[t]{2}{*}{ Dacriocito } & Perro & 223 & 54.4 \\
\hline & & Gato & 32 & 64.0 \\
\hline & \multirow[t]{2}{*}{ Codocito } & Perro & 28 & 6.8 \\
\hline & & Gato & 1 & 2.0 \\
\hline & \multirow[t]{2}{*}{ Estomatocito } & Perro & 2 & 0.5 \\
\hline & & Gato & 0 & 0.0 \\
\hline & \multirow[t]{2}{*}{ Eliptocito } & Perro & 1 & 0.2 \\
\hline & & Gato & 2 & 4.0 \\
\hline & Leptocito/ & Perro & 1 & 0.2 \\
\hline & Drepanocito & Gato & 1 & 2.0 \\
\hline & \multirow[t]{2}{*}{ Esquistocito } & Perro & 0 & 0.0 \\
\hline & & Gato & 1 & 2.0 \\
\hline & \multirow[t]{2}{*}{ Equinocito } & Perro & 0 & 0.0 \\
\hline & & Gato & 2 & 4.0 \\
\hline & \multirow[t]{2}{*}{ Eferocito } & Perro & 3 & 0.7 \\
\hline & & Gato & 1 & 2.0 \\
\hline \multirow{2}{*}{$\begin{array}{l}\text { Inclusión } \\
\text { intraeritrocitaria }\end{array}$} & \multirow{2}{*}{$\begin{array}{l}\text { Cuerpo de Howell- } \\
\text { Jolly }\end{array}$} & Perro & 1 & 0.2 \\
\hline & & Gato & 2 & 4.0 \\
\hline
\end{tabular}

FA: Frecuencia absoluta, FR: Frecuencia relativa

policromatofilia en el perro (Grindem et al., 1992). Sin embargo, los hallazgos de estos últimos autores no estuvieron presentes en los perros afectados con LMC. Por otra parte, aunque esta LMC en los gatos es de baja prevalencia, en este estudio se presentó en un $8 \%$, lo cual pudo estar condicionado por el tamaño de muestra utilizado en esta especie.
En el Cuadro 5 se evidencia el predominio de monocitosis con vacuolización citoplasmática en monocitos en los perros $(4.9 \%)$ y la trombocitosis con agregados plaquetarios en los gatos (12\%). Las causas principales de la monocitosis en perro son las lesiones inflamatorias (agudas o crónicas), procesos infecciosos, neoplasias, enfermeda- 
Cuadro 7. Análisis de frecuencia de variables de daño celular en las series mieloide y linfoide en perros $(\mathrm{n}=410)$ y gatos $(\mathrm{n}=50)$ con signos clínicos compatibles con afecciones hematológicas (Lima, Perú)

\begin{tabular}{llccc}
\hline Variable & Serie & Especie & FA & FR (\%) \\
\hline Núcleos atípicos en monocitos & Mieloide & Perro & 5 & 1.2 \\
& & Gato & 0 & 0 \\
Vacuolización citoplasmática en & & Perro & 60 & 14.6 \\
monocitos & Gato & 1 & 2.0 \\
Vacuolización citoplasmática en & & Perro & 2 & 0.5 \\
neutrófilos & Gato & 1 & 2.0 \\
Hiposegmentación nuclear en & Perro & 3 & 0.7 \\
neutrófilos & Gato & 0 & 0.0 \\
Cuerpo de Döhle en neutrófilos & Perro & 3 & 0.7 \\
& & Gato & 0 & 0 \\
Leucocitos pequeños (neutrófilos) & & Perro & 41 & 10.0 \\
& & Gato & 3 & 6.0 \\
Hipergranulación citoplasmática en & & Perro & 1 & 0.2 \\
eosinófilos & & Gato & 2 & 4.0 \\
Granulación tóxica en linfocitos & Linfoide & Perro & 1 & 0.2 \\
& & Gato & 0 & 0.0 \\
\hline
\end{tabular}

FA: Frecuencia absoluta, FR: Frecuencia relativa

des parasitarias, hemorragias, hemólisis y tratamiento con corticoesteroides, mientras que las causas fundamentales de la trombocitosis y la presentación de agregados paquetarios en gatos son las infecciones, los procesos inflamatorios y las neoplasias (Weiss y Wardrop, 2010). Es necesario mencionar que estas alteraciones de la serie mieloide estuvieron ausentes en los pacientes con LMC, pero presentes en los 13 animales observados con Anaplasma spp (perro), Mycoplasma spp (perro y gato) y Cytauxzoon felis (gato), junto a otros no infectados (Cuadro 8).

En el Cuadro 6 se exponen los principales hallazgos en la morfología celular de la serie eritroide en los perros y gatos evaluados. La principal alteración observada en el color eritrocítico en perros fue la policromatofilia (6.3\%), condición que estuvo presente en la anemia megaloblástica diagnosticada en esta especie (Cuadro 4, Figura 1).
La policromatofilia siempre que sea moderada, es un fenómeno fisiológico en la regeneración eritrocítica de la médula ósea, que está acompañado generalmente por un número significativo de macrocitos. Su apariencia ligeramente púrpura o violácea está determinada por la elevada concentración de ARN en los reticulocitos agregados y cuando esta concentración es baja es un indicativo de la presencia de reticulocitos punteados (Christian, 2010). Por otra parte, los cuerpos de Howell-Jolly son considerados inclusiones intraeritrocitarias, ya que son fragmentos nucleares o de nucléolos que aparecen en los eritrocitos maduros (Barger, 2010). Sin embargo, cuando estos son observados en sangre periférica es porque no han sido removidos del eritrocito al pasar por los sinusoides esplénicos (ausentes en los gatos) y con frecuencia se observan en anemias regenerativas y animales esplenectomizados (Barger, 2010). 
Cuadro 8. Análisis de frecuencia de microorganismos en eritrocitos en perros $(\mathrm{n}=410)$ y gatos $(\mathrm{n}=50)$ con signos clínicos compatibles con afecciones hematológicas (Lima, Perú)

\begin{tabular}{llccc}
\hline Microorganismo & Localización & Especie & FA & FR (\%) \\
\hline Anaplasma spp & Intracelular & Perro & 1 & 0.2 \\
& & Gato & 0 & 0.0 \\
Cytauxzoonfelis & & Perro & 0 & 0.0 \\
& & Gato & 10 & 20.0 \\
Mycoplasma spp & Extracelular & Perro & 1 & 0.2 \\
& & Gato & 1 & 2.0 \\
\hline
\end{tabular}

FA: Frecuencia absoluta, FR: Frecuencia relativa

Cuadro 9. Comparación de variables en la serie eritroide entre sexos en perros ( 189 hembras y 221 machos) y gatos (23 hembras y 27 machos) con signos clínicos compatibles con afecciones hematológicas (Lima, Perú)

\begin{tabular}{lccc}
\hline \multirow{2}{*}{ Variable } & \multirow{2}{*}{ Sexo } & \multicolumn{2}{c}{ Media } \\
\cline { 3 - 4 } & & Perro & Gato \\
\hline \multirow{2}{*}{ Hemoglobina $(\mathrm{g} / \mathrm{dl})$} & $\mathrm{H}$ & 14.00 & 12.73 \\
& $\mathrm{M}$ & 13.95 & 11.66 \\
Hematocrito $(\%)$ & $\mathrm{H}$ & 42.41 & 38.57 \\
& $\mathrm{M}$ & 42.24 & 35.33 \\
Eritrocitos $\left(\times 10^{6} / \mathrm{mm}^{3}\right)$ & $\mathrm{H}$ & 5.67 & 6.24 \\
& $\mathrm{M}$ & 5.65 & 5.89 \\
Porcentaje corregido de reticulocitos & $\mathrm{H}$ & 1.30 & 1.27 \\
Conteo absoluto de reticulocitos $-\mathrm{CAR}$ & $\mathrm{M}$ & 1.30 & 1.12 \\
$\left(\times 10^{3} / \mathrm{mm}^{3}\right)$ & $\mathrm{H}$ & 92.64 & 95.01 \\
Volumen corpuscular medio $-\mathrm{VCM}(\mathrm{fl})$ & $\mathrm{M}$ & 91.38 & 86.38 \\
Hemoglobina corpuscular media $-\mathrm{HCM}$ & $\mathrm{H}$ & 73.68 & 61.50 \\
$(\mathrm{pg})$ & $\mathrm{M}$ & 74.08 & 58.15 \\
Concentración de hemoglobina corpuscular & $\mathrm{H}$ & 24.32 & 20.28 \\
media - CHCM $(\mathrm{g} / \mathrm{dl})$ & $\mathrm{M}$ & 24.45 & 19.17 \\
\hline
\end{tabular}

Sin diferencias significativas entre sexos ( $p>0.05)$. Prueba de Wilcoxon

La clasificación del tamaño celular, según el VCM, evidenció una mayor incidencia de macrocitosis $(14.6 \%)$ y megalocitosis $(5.1 \%)$ en los perros en relación con los gatos (Cuadro 6). Ello infiere la liberación de formas inmaduras desde la médula ósea hasta la sangre periférica en eritropoyesis aceleradas para la compensación de determinadas anemias, aunque también pueden estar presentes en un diverso número de enfermedades (Weiss y Wardrop, 2010).
Por otra parte, se observaron con mayor frecuencia tres formas eritrocíticas anormales: los dacriocitos ( $64 \%$ en gato / $54.4 \%$ en perro), acantocitos ( $40 \%$ en gato $/ 11.7 \%$ en perro) y codocitos (6.8\% en perro / $2 \%$ en gato) (Cuadro 6). Los dacriocitos en los perros son el resultado del daño o distorsión de la membrana celular eritrocítica al pasar por los estrechos sinusoides medulares o esplénicos, y se presentan fundamentalmente en pacientes con desórdenes mielopro- 
Cuadro 10.Comparación de variables en las series mieloide y linfoide entre sexos en perros (189 hembras y 221 machos) y gatos (23 hembras y 27 machos) con signos clínicos compatibles con afecciones hematológicas (Lima, Perú)

\begin{tabular}{|c|c|c|c|}
\hline \multirow{2}{*}{ Variable } & \multirow{2}{*}{ Sexo } & \multicolumn{2}{|c|}{ Media } \\
\hline & & Perro & Gato \\
\hline \multirow[t]{2}{*}{ Leucocitos $\left(\mathrm{x} 10^{3} / \mathrm{mm}^{3}\right)$} & $\mathrm{H}$ & 14.38 & 18.03 \\
\hline & M & 14.65 & 15.59 \\
\hline \multirow[t]{2}{*}{ Precursores linfoides (\%) } & $\mathrm{H}$ & 0.22 & 0.13 \\
\hline & M & 0.19 & 0.37 \\
\hline \multirow[t]{2}{*}{ Precursores mieloides (\%) } & $\mathrm{H}$ & 0.31 & 0.04 \\
\hline & M & 0.06 & 0 \\
\hline \multirow{2}{*}{ Neutrófilos bandas (\%) } & $\mathrm{H}$ & 0.50 & 0.57 \\
\hline & M & 0.62 & 0.19 \\
\hline \multirow[t]{2}{*}{ Neutrófilos segmentados (\%) } & $\mathrm{H}$ & 72.63 & 64.61 \\
\hline & M & 72.29 & 70.48 \\
\hline \multirow[t]{2}{*}{ Eosinófilos (\%) } & $\mathrm{H}$ & $2.11^{\mathrm{a}}$ & 5.17 \\
\hline & M & $2.70^{\mathrm{b}}$ & 3.81 \\
\hline \multirow[t]{2}{*}{ Basófilos (\%) } & $\mathrm{H}$ & 0.05 & 0.09 \\
\hline & M & 0.04 & 0.07 \\
\hline \multirow[t]{2}{*}{ Monocitos (\%) } & $\mathrm{H}$ & 5.37 & 3.74 \\
\hline & M & 5.56 & 3.11 \\
\hline \multirow[t]{2}{*}{ Linfocitos $(\%)$} & $\mathrm{H}$ & 18.83 & 25.65 \\
\hline & M & 18.54 & 21.96 \\
\hline \multirow[t]{2}{*}{ Plaquetas $\left(\mathrm{x} 10^{3} / \mathrm{mm}^{3}\right)$} & $\mathrm{H}$ & $439.17^{\mathrm{a}}$ & 598.00 \\
\hline & M & $493.25^{\mathrm{b}}$ & 623.22 \\
\hline
\end{tabular}

a,b Superíndices diferentes entre sexos dentro de una variable indican diferencia significativa $(p<0.05)$.

Prueba de Wilcoxon

liferativos o con hiperesplenismo (Barger, 2010). A diferencia de lo anterior, los acantocitos en los perros aparecen mayormente en enfermedades hepáticas crónicas, desviación portosistémica, CID, neoplasias y nefropatías (glomerulonefritis), mientras que en los gatos es la forma eritrocitaria anormal más común y presente en enfermedades hepáticas (Barger, 2010). Sin embargo, este autor señala que los codocitos emergen principalmente en las anemias regenerativas y por deficiencia de hierro, enfermedades hepáticas, así como en animales esplenectomizados.

En contraste a lo anterior, los cambios anormales en la morfología celular en las series mieloide y linfoide presentes en el Cuadro 7 son más concretas en las dos especies estudiadas. Aquí se evidenció que la vacuolización citoplasmática en monocitos y la existencia de leucocitos pequeños (neutrófilos) tuvieron mayor frecuencia de presentación, tanto en perros como gatos, además de la hipergranulación citoplasmática en eosinófilos observada en los felinos.

Según Weiss y Wardrop (2010), la vacuolización citoplasmática en monocitos es el cambio más frecuente en los monocitos activados. Estos cambios están asociados fundamentalmente a infecciones (víricas y bacterianas), procesos inflamatorios o necróticos, neoplasias, y corticoesteroides. Sin embargo, la observación de neutrófilos maduros de pequeño tamaño está presente en la mayoría de las leucemias mieloides crónicas (Dobson et al., 2006; Weiss y Wardrop, 


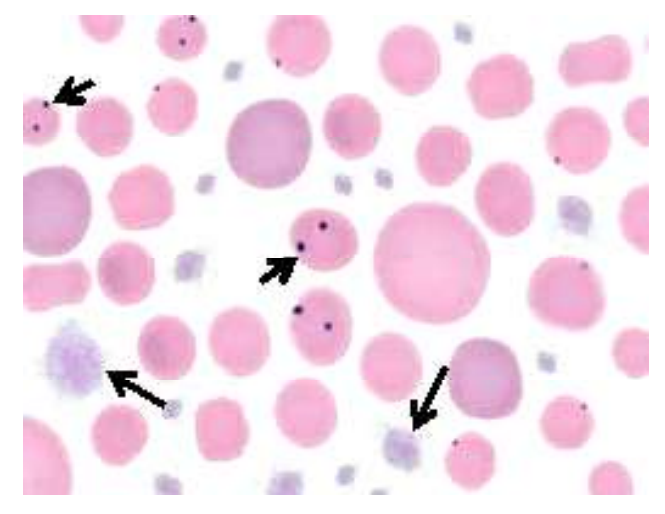

Figura 3. Anaplasmosis en perro. Anaplasma spp intraeritrocitario (flecha corta) y abundantes macroplaquetas (flecha larga). Se observan abundantes megalocitos, anisocitosis y policromasia. Tinción de Wright (100X)

2010). En contraste, según estos últimos autores, la función primaria de los eosinófilos en los gatos y otras especies es la destrucción de parásitos y la modulación de las reacciones de hipersensibilidad. Tanto la presencia de vacuolas en monocitos como la hipergranulación en eosinófilos responden principalmente a patrones infecciosos en los animales afectados en este estudio.

Las prevalencias en los perros de organismos eritrocitarios compatibles con Anaplasma spp y Mycoplasma spp observadas fueron bastante bajas (Cuadro 8), habiendo solo un perro positivo en cada caso. Sin embargo, se observó Cytauxzoon felis en 10 gatos (20\%) y Mycoplasma spp en el $2 \%$ de los felinos (1/50). En la Figura 3 se observa un frotis con Anaplasma spp en un perro con anemia megaloblástica y trombocitosis severa.

La anaplasmosis es una enfermedad infrecuente en los gatos; sin embargo, en los perros es común la transmisión de $A$. platys por picaduras de Rhipicephalus spp y de $A$. phagocytophilum por Ixodes spp (De
Tommasi et al., 2014; El Hamiani-Khatat et al., 2015). En el perro, A. platys desencadena monocitosis y severa trombocitopenia (replicación intraplaquetaria) (De Tommasi et al., 2014) у A. phagocytophilum ocasiona anemia severa normocítica hipocrómica regenerativa, ligera leucocitosis con neutrofilia, trombocitopenia y monocitosis (El Hamiani-Khatat et al., 2015; Lappin et al., 2015). En los gatos, A. phagocytophilum produce, además de la anemia, linfopenia, neutrofilia o neutropenia (Savidge et al., 2016). Este género ha sido mencionado como causa de determinadas leucemias mieloides agudas en perros, condición que cobra un importante valor en el diagnóstico clínico veterinario (Bezerra et al., 2018). La prevalencia de estas rickettsias es relativamente baja en los perros (20\%) (El Hamiani-Khatat et al., 2017), lo cual coincide con el bajo valor obtenido en este estudio (Cuadro 8) y probablemente justifica los escasos reportes publicados en el país.

Cytauxzoon felis es un protozoo intracelular con reservorio natural en los felinos silvestres y sus vectores lo constituyen las garrapatas de los géneros Amblyomma, Dermacentor e Ixodes (Shock et al., 2014). La prevalencia de $C$. felis en gatos es baja, reportándose $0.7 \%$ en Brasil (André et al., 2015) y $1.2 \%$ en España (Díaz-Regañón et al., 2017). Spada et al. (2014) observaron anemia (46\%), leucopenia (9.3\%), leucocitosis $(3.3 \%)$ y trombocitopenia $(6.7 \%)$ en gatos infectados con C. felis, mientras que Legroux et al. (2017) observaron moderada leucocitosis, neutrofilia, linfocitosis y eosinofilia. Sin embargo, en este estudio su prevalencia fue superior, dando un 20\% (Cuadro 8), lo que quizás pudo estar determinado por la presencia de estos vectores en su entorno. En la Figura 4 se evidencia la presencia de C. felis intraeritrocitario, policromasia, poiquilocitosis, anisocitosis y trombocitosis, $\mathrm{y}$ en la Figura 5 se observa la muestra de perro positiva a Mycoplasma spp. 


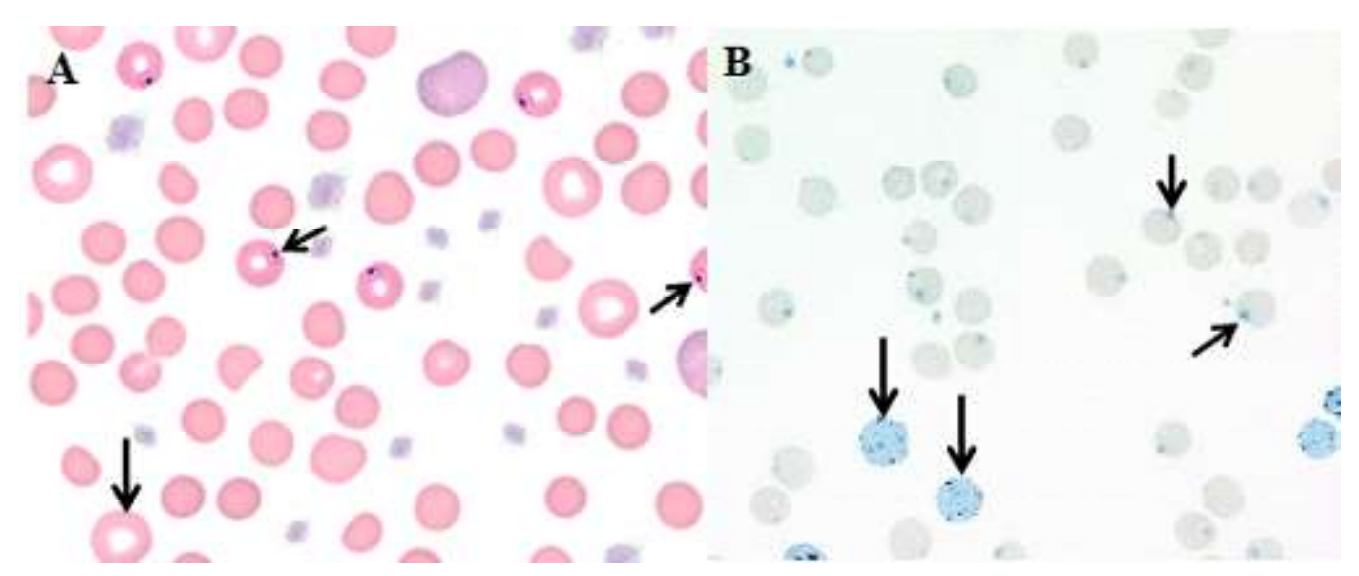

Figura 4. Cytauxzoonosis en gato. A: Cytauxzoon felis intraeritrocitario (flecha corta). Poiquilocitosis, abundantes eferocitos y leptocitos (flecha larga). Megalocitos con hipercromasia. Trombocitosis con macroplaquetas. Tinción de Wright (100X). B: Observación en paralelo de $C$. felis en el espacio intracelular de eritrocitos (flecha corta) con abundantes reticulocitos (flecha larga). Tinción Azul de cresil brillante (100X)

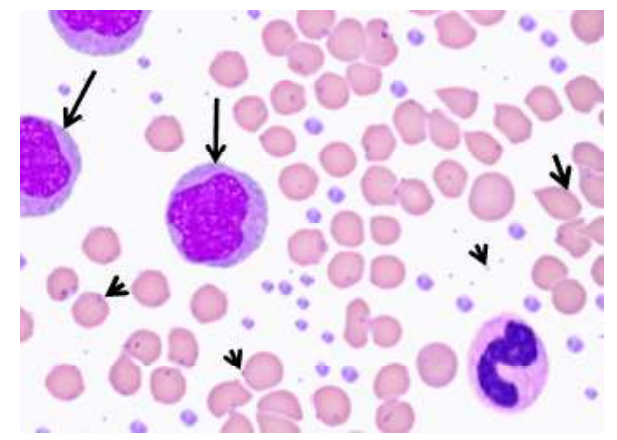

Figura 5. Micoplasmosis en perro. Mycoplasma spp en la superficie eritrocitaria y fuera de esta (cabeza de flecha). Abundantes dacriocitos (flecha corta), y severas monocitosis (flecha larga) y trombocitosis (con tendencia a formar agregados). Tinción de Wright (100X)

La baja prevalencia de Mycoplasma spp en este estudio (Cuadro 8) coincide con el reporte de $3.7 \%$ de Mycoplasma haemofelis y $14.3 \%$ de Mycoplasma haemocanis en España (Roura et al., 2010), así como con el 4 y $4.5 \%$ en Italia para estos microorganismos, respectivamente (Ravagnan et al., 2017). Asimismo, Hasiri et al. (2016) reportaron una frecuencia de 7.5\% de $M$. haemocanis en perros de Irán, la que estuvo acompañada de anemia (11.5\%), leucocitosis y trombocitosis. Resulta importante añadir que $M$. haemofelis ha sido causa de leucemia mieloide aguda en gatos domésticos (Maazi et al., 2013), y en algunos casos de meningoencefalitis, acompañada de leucocitosis, neutrofilia y monocitosis (Beauchamp et al., 2011). Probablemente los dos casos encontrados en el presente estudio sean de animales inmunodeprimidos, por ser esta una de las condiciones predisponentes más comunes en la micoplasmosis canina y felina (Allison y Meinkoth, 2010).

Llama la atención en este estudio que casi todos los gatos y perros infectados con estos microorganismos presentaron monocitosis y trombocitosis (Figuras 3-5), cuando la mayoría de los estudios reportan comúnmente la trombocitopenia y neutrofilia (Beauchamp et al., 2011; Spada et al., 2014; Lappin et al., 2015; Legroux et al., 2017). 
En el Cuadro 9 se muestra la comparación de medias entre sexos de las variables en la serie eritroide en ambas especies, no evidenciándose diferencias estadísticas significativas según la prueba de Wilcoxon. La comparación de medias en las series mieloide y linfoide entre sexos de perros y gatos solo evidenciaron diferencias significativas en las plaquetas totales $(\mathrm{p}=0.0087$ ) y los eosinófilos $(p=0.0260)$ en los perros machos (Cuadro 10). No se evidenció diferencia alguna en el resto de las variables analizadas por la prueba de Wilcoxon, aunque la trombocitosis estuvo presente en los perros evaluados.

Se concluye que la prevalencia de Cytauxzoon felis en los gatos estudiados es relativamente baja y existe un riesgo de zoonosis de Anaplasma spp y Mycoplasma spp en los propietarios de animales afectados en Lima, Perú.

\section{Limeratura Citada}

1. Allison RW, Meinkoth JH. 2010. Anemia caused by rickettsia, Mycoplasma, and protozoa. In: Weiss DJ, Wardrop JK (eds). Schalm's veterinary hematology. $6^{\text {th }}$ ed. USA: Wiley-Blackwell. p 199-210.

2. Allison RW, Little SE. 2013. Diagnosis of rickettsial diseases in dogs and cats. Vet Clin Path 42: 127-144. doi: 10.1111/ vcp. 12040

3. André MR, Herrera HM, Fernandes SJ, de Sousa KC, Gonçalves LR, Domingos IH, de Macedo GC, et al. 2015. Tick-borne agents in domesticated and stray cats from the city of Campo Grande, state of Mato Grosso do Sul, midwestern Brazil. Ticks Tick-Borne Dis 6: 779-786. doi: 10.1016/j.ttbdis.2015.07.004

4. Barger AM. 2010. Erythrocyte morphology. In: Weiss DJ, Wardrop JK (eds). Schalm's veterinary hematology. $6^{\text {th }}$ ed. USA: Wiley-Blackwell. p 144-151.
5. Beauchamp DJ, da Costa RC, Premanandan C, Burns CG, Cui J, Daniels JB. 2011. Mycoplasma felisassociated meningoencephalomyelitis in a cat. J Feline Med Surg 13: 139-143. doi: 10.1016/j.jfms.2010.10.004

6. Bezerra JAB, Rodrigues RTGA, Lima IO, Vale AM, Filgueira KD. 2018. Acute myeloid leukemia in a dog chronically infected with Leishmania spp and other infectious agents. Acta Sci Vet 46(Suppl 1): 260. doi: 10.22456/16799216.85945

7. Bustamante R. 2015. Frecuencia de alteraciones eritrocitarias en perros (Canis lupus familiaris) registradas en el Laboratorio Clínico Veterinario CEDIVETLima. Tesis de Médico Veterinario. Lima: Univ. Nacional de Piura. $61 \mathrm{p}$.

8. Christian JA. 2010. Erythrokinetics and erythrocyte destruction. In: Weiss DJ, Wardrop JK (eds). Schalm's veterinary hematology. $6^{\text {th }}$ ed. USA: WileyBlackwell. p 136-143.

9. De Tommasi AS, Baneth G, Breitschwerdt EB, Stanneck D, Dantas-Torres $F$, Otranto $D$, de Caprariis $D$. 2014. Anaplasma platys in bone marrow megakaryocytes of young dogs. J Clin Microbiol 52: 2231-2234. doi: 10.1128/JCM.00395-14

10. Díaz-Regañón D, Villaescusa A, Ayllón T, Rodríguez-Franco F, Baneth G, Calleja-Bueno L, García-Sancho M, et al. 2017. Molecular detection of Hepatozoon spp and Cytauxzoon sp in domestic and stray cats from Madrid, Spain. Parasite Vector 10: 112. doi: 10.1186/s13071-017-2056-1

11. Dobson J, Villiers E, Morris J. 2006. Diagnosis and management of leukaemia in dogs and cats. In Practice 28: 22-31. doi: 10.1136/inpract.28.1.22

12. El Hamiani-Khatat $S$, Daminet $S$, Kachani $M$, Leutenegger $C M$, Duchateau L, El Amri H, Hing M, et al. 2017. Anaplasma spp in dogs and owners in north-western Morocco. Parasite Vector 10: 202. doi: 10.1186/ s13071-017-2148-y 
13. El Hamiani-Khatat S, Defauw P, Marynissen S, Van de Maele I, van Dongen A, Daminet S. 2015. Exposure to Anaplasma phagocytophilum in two dogs in Belgium. Vlaams Diergen Tijds 84: 39-46.

14. Ewing P. 2018. Iron deficiency in dogs and cats. [Internet]. Available in: https:// www.mspca.org/angell_services/irondeficiency-in-dogs-and-cats/

15. Fine DM, Tvedten HW. 1999. Chronic granulocytic leukemia in a dog. J Am Vet Med Assoc 214: 1809-1812.

16. Forrellat-Barrios M, HernándezRamírez P, Fernández-Delgado N, Pita-Rodríguez G. 2010. ¿Se cumple siempre la relación hemoglobinahematocrito? Rev Cubana Hematol Inmunol Hemoter 26: 359-361.

17. Gholipour B. 2016. These 7 foods cause the most pet deaths. LiveScience. [Internet]. Available in: https://www.livescience.com/54860-foods-cause-themost-pet-deaths.html

18. Grindem CB, Stevens JB, Brost DR, Johnson DD. 1992. Chronic myelogenous leukaemia with meningeal infiltration in a dog. Comp Haematol Int 2: 170-174. doi: 10.1007/BF00426173

19. Hasiri MA, Sharifiyazdi H, Moradi T. 2016. Molecular detection and differentiation of canine hemoplasma infections using RFLP-PCR in dogs in southern Iran. Vet Arhiv 86: 529-540.

20. Lappin MR, Chandrashekar R, Stillman B, Liu J, Mather TN. 2015. Evidence of Anaplasma phagocytophilum and Borrelia burgdorferi infection in cats after exposure to wildcaught adult Ixodes scapularis. J Vet Diagn Invest 27: 522-525. doi: 10.1177/ 1040638715593598

21. Legroux JP, Halos L, René-Martellet $M$, Servonnet $M$, Pingret $J L$, Bourdoiseau G, Baneth G, et al. 2017. First clinical case report of Cytauxzoon sp infection in a domestic cat in France. BMC Vet Res 13: 81. doi: 10.1186/ s12917-017-1009-4
22. Maazi N, Nassiri SM, Nemati G, Mirlatifi S, Firouzbakht F. 2013. Case report: diagnosis of acute monocytic leukaemia (AML-M5b) in a juvenile cat. Rev Med Vet-Toulouse 164: 358-361.

23. Manual Veterinario de Coagulación. 2018. Laboratorio Suiza Vet. Lima, Perú. [Internet]. Disponible en: http:// www.suizavet.com/manuales/ coagulacion.pdf

24. Manual Veterinario de Hematología. 2018. Laboratorio Suiza Vet, Lima, Perú. [Internet]. Disponible en: http:// www.suizavet.com/manuales/ hematologia.pdf

25. Marino CL, Tran JNSN, Stokol T. 2017. Atypical chronic myeloid leukemia in a German Shepherd dog. J Vet Diagn Invest 29: 338-345. doi: 10.1177/ 1040638716689581

26. Muñoz MZ, Morón CC. 2005. Manual de procedimientos de laboratorio en técnicas básicas de hematología. Lima, Perú: Instituto Nacional de Salud, Ministerio de Salud. $88 \mathrm{p}$.

27. Palmer L, Briggs C, McFadden S, Zini G, Burthem J, Rozenberg G, Proytcheva M, et al. 2015. ICSH recommendations for the standardization of nomenclature and grading of peripheral blood cell morphological features. Int J Lab Hematol 37: 287-303. doi: 10.1111/ijlh.12327

28. Palomo IG, Cruzat CC, Alarcón ML, Agurto MO, Retamales EC. 2005. Hemograma, mielograma y biopsia de médula ósea. En: Palomo IG, Pereira JG, Palma JB (eds). Hematología: fisiopatología y diagnóstico. Chile: Univ. de Talca. $666 \mathrm{p}$.

29. Pérez NC. 2016. Manual práctico. Hospital Veterinario de Pequeñas Especies. Universidad Veracruzana. [Internet]. Disponible en: http://conevet.org.mx/ appvisitas2013/public/uploads/ $29 \_9 \_14 \quad 3 . p d f$

30. Ramis $\overline{J B}$. 2014. Interpretación del hemograma pediátrico. [Internet]. Disponible en: https://www.parcde-salut- 
mar.cat/mar/interpreta-cion $\% 20$ hemograma_2013.pdf

31. Ravagnan $\bar{S}$, Carli E, Piseddu E, Da Rold G, Porcellato E, Zanardello C, Carminato A, et al. 2017. Prevalence and molecular characterization of canine and feline hemotropic mycoplasmas (hemoplasmas) in northern Italy. Parasite Vector 10: 132. doi: 10.1186/s13071-0172069-9

32. Reagan WJ, Irizarry ARR, DeNicola DB. 2019. Veterinary hematology: atlas of common domestic and nondomestic species. $3^{\text {rd }}$ ed. USA: John Wiley \& Sons. $122 \mathrm{p}$.

33. Rizzi TE, Meinkoth JH, Clinkenbeard KD. 2010. Normal hematology of the dog. In: Weiss DJ, Wardrop JK (eds). Schalm's veterinary hematology. $6^{\text {th }} \mathrm{ed}$. USA: Wiley-Blackwell. p 799-810.

34. Roura X, Peters IR, Altet L, Tabar MD, Barker EN, Planellas M, Helps CR, et al. 2010. Prevalence of hemotropic mycoplasmas in healthy and unhealthy cats and dogs in Spain. J Vet Diagn Invest 22: 270-274. doi: 10.1177/104063871002200219

35. Savidge C, Ewing P, Andrews J, Aucoin D, Lappin M, Moroff S. 2016. Anaplasma phagocytophilum infection of domestic cats: 16 cases from the northeastern USA. J Feline Med Surg 18: 85-91. doi: 1098612X15571148

36. Shock BC, Moncayo A, Cohen S, Mitchell EA, Williamson PC, Lopez, G, Garrison LE, et al. 2014. Diversity of piroplasms detected in blood-fed and questing ticks from several states in the United States. Ticks Tick-Borne Dis 25: 373-380. doi: 10.1016/j.ttbdis.2014.01.003

37. Spada E, Proverbio D, Galluzzo P, Perego R, De Giorgi GB, Roggero N, Caracappa S. 2014. Frequency of piroplasms Babesia microti and Cytauxzoon felis in stray cats from northern Italy. Biomed Res Int 2014: 943754. doi: 10.1155/2014/943754

38. Swann JW, Szladovits B, Glanemann B. 2016. Demographic characteristics, survival and prognostic factors for mortality in cats with primary immunemediated hemolytic anemia. J Vet Intern Med 30: 147-156. doi: 10.1111/jvim.13658

39. Weiss DJ, Wardrop JK. 2010. Schalm's veterinary hematology. $6^{\text {th }}$ ed. Iowa, USA: Wiley-Blackwell. 1206 p. 